\title{
Assessment of a Low Profile Planar Antenna for a Wireless Sensor Network Monitoring the Local Water Distribution Network
}

This paper is a postprint of a paper submitted to and accepted for publication in IET Wireless Sensor Systems and is subject to Institution of Engineering and Technology copyright. The copy of record is available at IET Digital Library 


\title{
Assessment of a Low Profile Planar Antenna for a Wireless Sensor Network Monitoring the Local Water Distribution Network.
}

\author{
Steven Herbert, Tian-Hong Loh (member IET), Ian Wassell (member IET)
}

October 14, 2014

\begin{abstract}
This paper presents an assessment on the suitability of a low-profile planar antenna for a Wireless Sensor Network (WSN) application monitoring the water supply at Fire Hydrants (FHs). The antenna must have a low profile so that it can be mounted on the FH lid; it must have an omnidirectional radiation pattern so that it can communicate with base stations at low elevations; and it must operate in the $2.4 \mathrm{GHz}$ Industrial, Scientific and Medical (ISM) band. Measurements show that for the majority of the $2.4 \mathrm{GHz}$ ISM band, the antenna has a return loss of at least $-10 \mathrm{~dB}$ and efficiency greater than $60 \%$.
\end{abstract}

For the FH WSN assessment, the antenna was deployed as a transmitter mounted on the FH lid above the underground FH chamber and a vertically polarised monopole antenna mounted on a mast at various specified heights above ground level was used to measure the received power as a function of distance. The path loss results were compared with those from a previous deployment, where the FH antenna was located in the FH chamber, and it is found that using the low-profile antenna reduced the path loss by at least $10 \mathrm{~dB}$ over the measured transmitter and receiver separation.

\section{Introduction}

It has previously been identified that an important application for Wireless Sensor Networks (WSNs) is monitoring the pressure, acoustic noise, turbidity and flow rate of the local water distribution network via sensors located within Fire Hydrant $(\mathrm{FH})$ chambers [1-3]. In the FH 


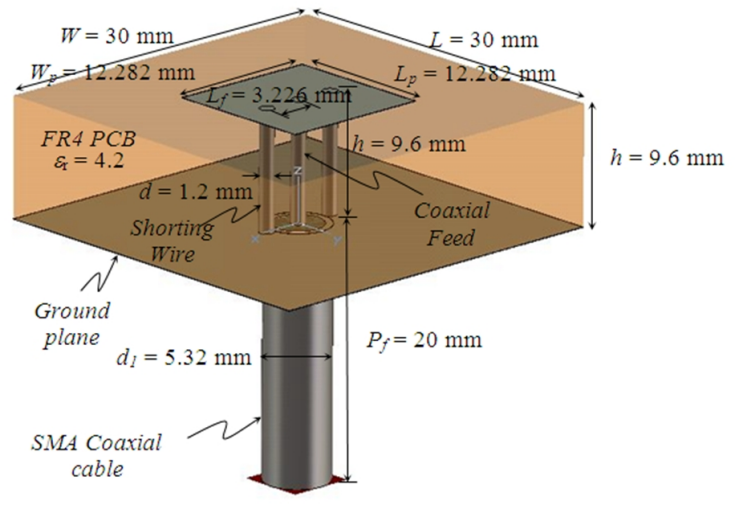

Figure 1: Printed patch-type antenna.

application the antenna must communicate with above ground base stations (BSs) mounted on nearby buildings (i.e., at low elevations above the horizontal). Current civil FH water supply infrastructure has located the wireless sensor node antenna within the below ground $\mathrm{FH}$ chambers that have lids made of cast iron [1] which results in poor communication with the above ground BS (owing to the metal components within the chamber, and its assymetric layout mean it is unlikely that the FH will have an omnidirectional radiation pattern). Therefore we propose that it would be desirable to have a low-profile over-ground antenna mounted on the cast iron lid to address this problem. Lin et al [1] also identify the $2.4 \mathrm{GHz}$ Industrial Scientific and Medical (ISM) as the prefered band of operation, and we are especially interested in the performance between 2.4 and $2.48 \mathrm{GHz}$, i.e., the band covering the 802.15.4 channels [4] used by many WSNs such as the Memsic MICAz [5].

We therefore specify three requirements for the antenna: firstly that it must be low profile, so we can mount it on the FH lid; secondly it must have an omnidirectional radiation pattern, so that it can communicate effectively to the low elevation BSs; and thirdly it must operate between 2.4 and $2.48 \mathrm{GHz}$. High profile omnidirectional antennas such as monopoles, oriented for uniform radiation in horizontal directions on the surface of the Earth are widely used for terrestrial wireless communication networks. However, they are not suitable for the aforementioned WSN monitoring applications due to their high profile nature. Hence, simple planar antennas with monopole-like vertically polarised omnidirectional radiation patterns are ideal for this ap- 


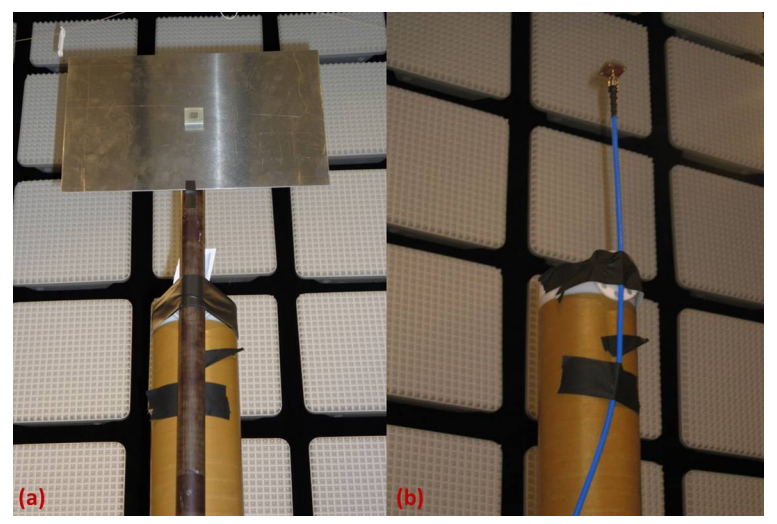

Figure 2: (a): Printed patch-type antenna with antenna stood $1 \mathrm{~mm}$ off ground plane; (b) Printed patch-type antenna without ground plane.

plication. To this end, we have identified a low-profile printed patch-type antenna, based on those proposed by Delaveaud et al [6]; Conway et al [7], [8]; and Chandran et al [9] as the ideal candidate. The developed printed patch-type antenna is shown in Figure 1; it operates between $2.4-2.48 \mathrm{GHz}$ and consists of a ground plane and patch metallisation on a dielectric substrate, a coaxial feed probe connected to the centre of the patch through the ground plane, a dielectric substrate, and two ground shorting vias offset from the feed. The antenna shorting via placements have been optimised for operation above a metallic ground plane having the same dimensions as the FH lid (see Figure 2 (a)), and the chosen dielectric is FR4, so that it can be manufactured using standard PCB processing methods. In this work, we aim to assess the effectiveness of our antenna for the FH application.

The remainder of the paper is organised as follows: In Section II we characterise the antenna and compare the result to the simulations undertaken during the design process; in Section III we assess the effectiveness of the antenna when deployed at the FH; and finally conclusions are drawn in Section IV.

\section{Characterisation of the Antenna}

The objective of the characterisation is to confirm that the antenna operates between 2.4 and $2.48 \mathrm{GHz}$ with the desired omnidirectional radiation pattern and with high efficiency. The mea- 


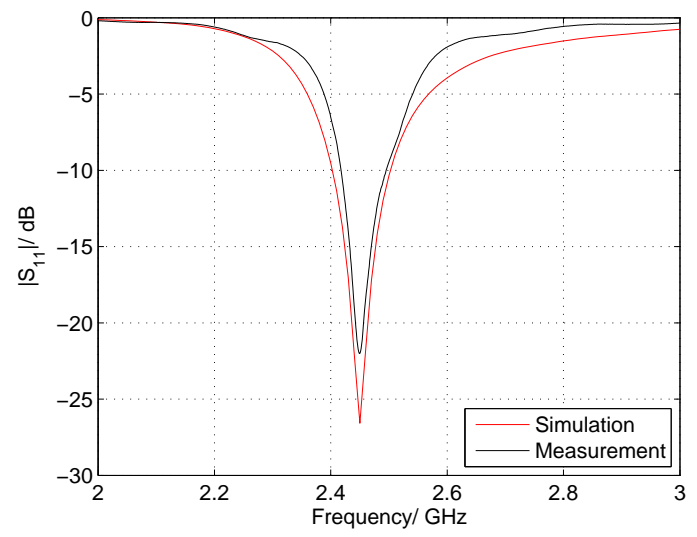

Figure 3: $\left|S_{11}\right|$ of patch antenna without ground plane.

surements were undertaken in the UK National Physical Laboratory (NPL) Small Antenna Radiated Testing (SMART) range [10], [11] and compared to simulations performed using CST Microwave Studio [12]. The antenna design has taken into account that when deployed at the FH it will be mounted on an additional ground plane (see Figure 6-(c)), therefore the characterisation takes place both with and without the ground plane, as shown in Figure 2. Figure 3 shows the $S_{11}$ characterisation of the antenna, and we observe a reasonable agreement between simulation and measurement results. We require that the return loss is at least $-10 \mathrm{~dB}$ [13], and we can see that this is the case for the majority of the required operating band $(2.42-2.48 \mathrm{GHz})$.

To calculate the radiation efficiency we performed a three dimensional radiation pattern measurement of the antenna in the SMART range to find the antenna directivity (we then measure the antenna gain using substitution method with a reference gain antenna and finally evaluate the efficiency with the measured directivity and gain). We see in Figure 4 that the efficiency is between $60 \%$ and $73 \%$ throughout the band, which is acceptable for most wireless applications.

Figures 5 (a) and 5 (b) show the E-plane cuts for the antenna both with and without ground plane respectively. We note that the ground plane has the effect of 'pushing' the direction of maximum radiation away from the horizontal to approximately $40^{\circ}$ above the horizontal. We can also see that there is some discrepancy between simulation and measurements for the case 


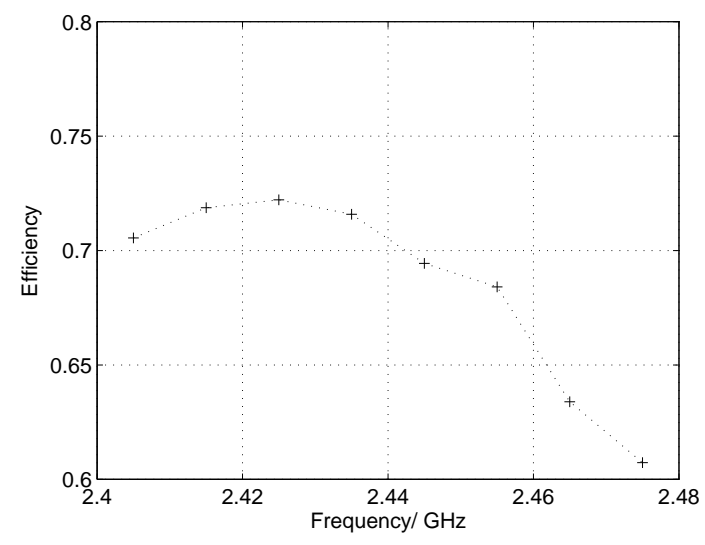

Figure 4: Antenna radiation efficiency without ground plane.

without the ground plane, however with the ground plane in place the agreement is very good. A possible explanation for this is that the ground plane reduces the effect of the cable, which was not included in the simulation.

It is required that when deployed at the FH (i.e., mounted on the ground plane) the antenna radiation pattern is omnidirectional. We can see from the H-plane cut shown in Figure 5 (d) that this is indeed the case. Figure 5 (c) shows that the radiation pattern is also omnidirectional without the ground plane.

\section{Fire Hydrant WSN Assessment}

We undertook propagation measurements to evaluate the performance of the low profile antenna in the actual FH water supply infrastructure. Figure 6 shows the measurement set-up. Note that this shows a prototype only, and for a finished product the problem of how to integrate the antenna such that its protrusion does not cause practical problems (such as tripping) must be addressed. The aim is to measure the received signal power as a function of separation between a vertically pointing receiving half wavelength dipole antenna located at various specified heights above ground level and the designed transmitting low profile antenna located on the lid of the FH. The receive antenna is mounted at heights of $2.14 \mathrm{~m}, 4.14 \mathrm{~m}$ and $6.29 \mathrm{~m}$ above ground level and the received signal power measurements were recorded while pushing the trolley at an 


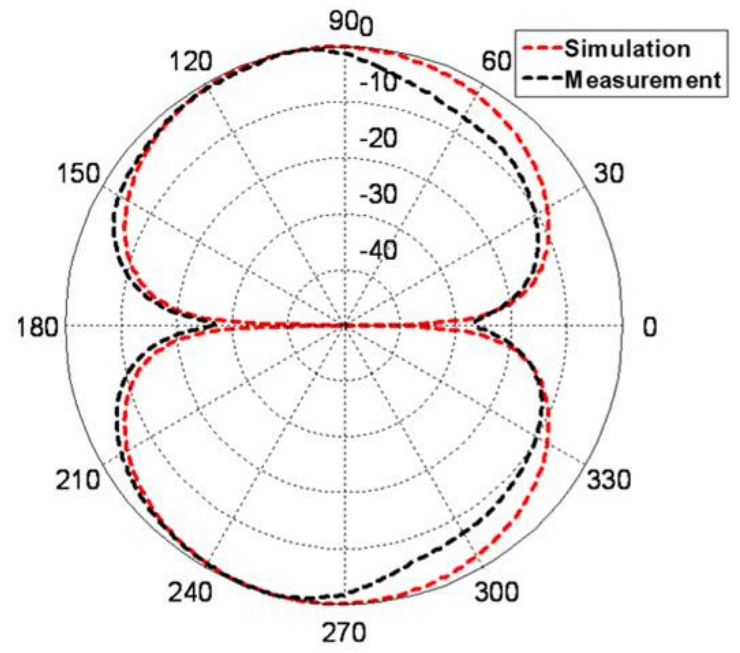

(a)

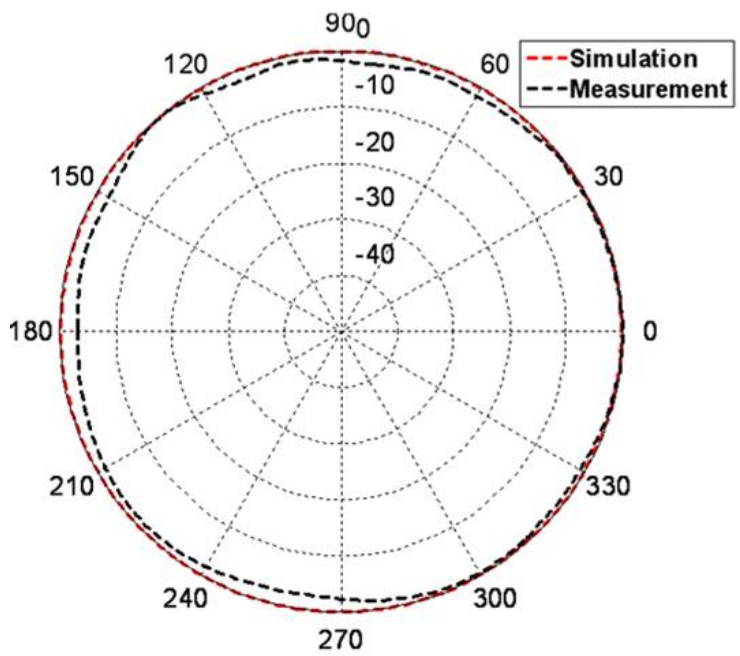

(c)

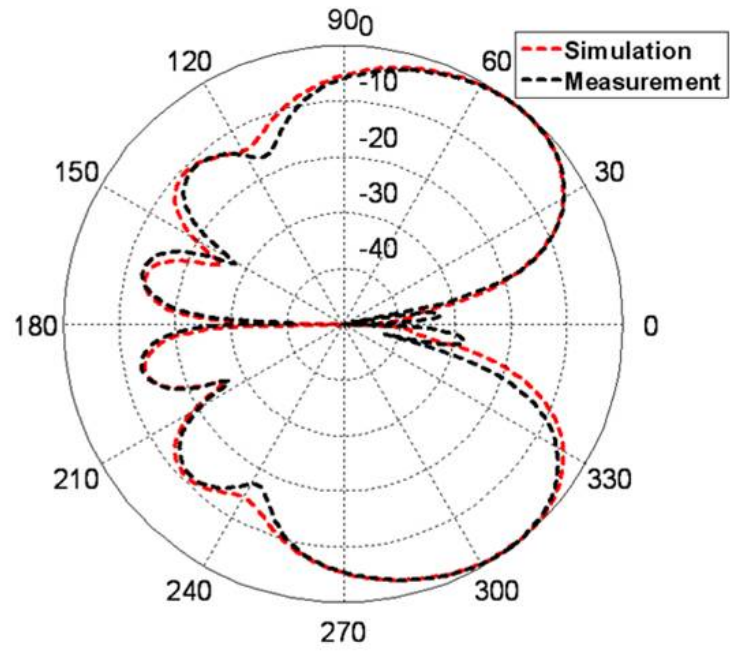

(b)

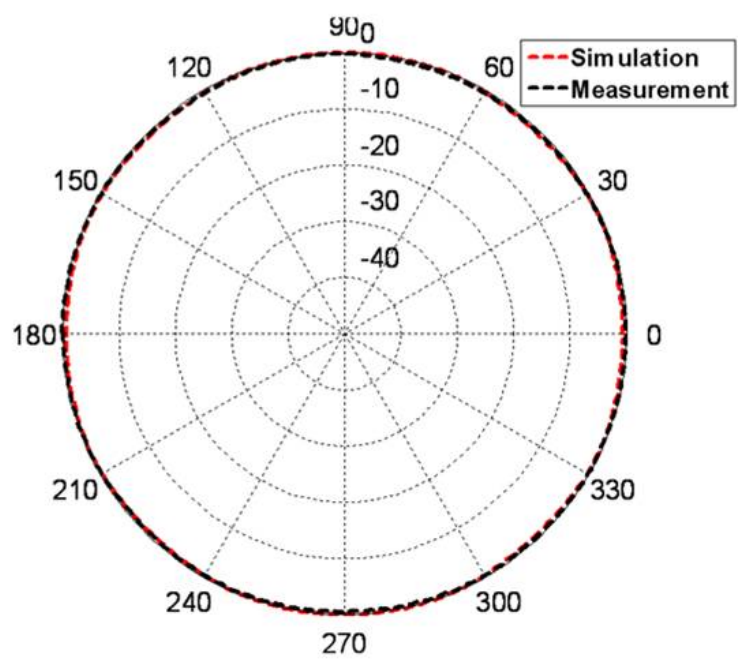

(d)

Figure 5: (a) E-plane co-polar cut without the metallic ground plane; (b) E-plane co-polar cut with the metallic ground plane, antenna stand-off $1 \mathrm{~mm}$; (c) H-plane co-polar cut without the metallic ground plane; (d) H-plane co-polar cut with the metallic ground plane, antenna stand-off $1 \mathrm{~mm}$;

approximately constant velocity. Note that the trolley velocity is low (slow walking pace) and the purpose of moving the trolley is to characterise the propagation between the FH and a stationary $\mathrm{BS}$ as a function of distance (as opposed to between the $\mathrm{FH}$ and a mobile antenna). To investigate the effectiveness of reducing the $\mathrm{FH}$ antenna peak gain angle, two transmit antenna 


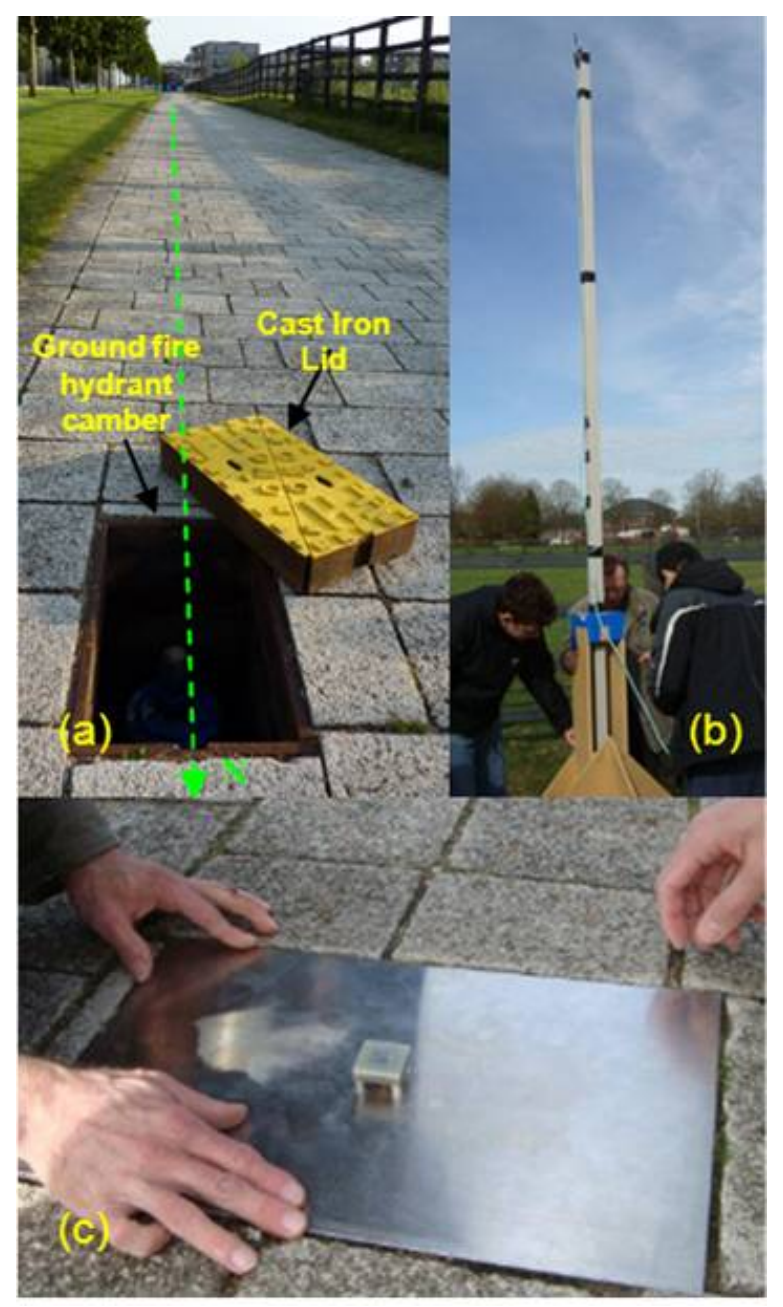

Figure 6: (a): The fire hydrant measurement environment; (b)- the experimental set-up at the receiving antenna on a trolley mounted mast; (c)- the designed transmitting low profile antenna mounted on a metallic ground plane that replaces the original cast iron FH lid.

configurations are studied, namely, $1 \mathrm{~mm}$ stand-off (i.e., the antenna mounted directly on the metallic ground plane) or $10 \mathrm{~mm}$ stand-off (i.e., the antenna $10 \mathrm{~mm}$ above the metallic ground plane). The transmitter unit comprises a battery powered signal source operating at $2.485 \mathrm{GHz}$ (note that this is marginally outside of the specified $2.4-2.48 \mathrm{GHz}$ range, however this is not crucial).

From the flat-earth model, we expect to see a slope with gradient of $-40 \mathrm{~dB} /$ decade of received 
signal with distance, at sufficiently large distances from the transmitter [14]:

$$
\left(\frac{P_{R}}{P_{T}}\right)=G_{T} G_{R}\left(\frac{h_{T} h_{R}}{d^{2}}\right)^{2}
$$

where $P_{R}$ and $P_{T}$ are the received and transmitted powers, respectively, $G_{R}$ and $G_{T}$ are the receive and transmit antenna gains, respectively, $h_{R}$ and $h_{T}$ are the receive and transmit antenna heights above the plane, respectively and $d$ is the separation distance between the transmitter and receiver.

The path loss $(P L)$ is defined as in [1]:

$$
P L_{(d B)}=P_{T(d B m)}+G_{T(d B)}+G_{R(d B)}-P_{R(d B m)} .
$$

We set $G_{T(d B)}=0$, i.e., we treat the gain of the low-profile antenna as zero, as we are interested in its performance as part of the channel (i.e., contributing to the path loss), we use the standard dipole gain of $1.76 \mathrm{dBi}$ for the receive antenna gain.

Plots of path loss as a function of distance are shown in Figure 7 (a) and (b) for $1 \mathrm{~mm}$ and $10 \mathrm{~mm}$ stand-offs respectively with a receiver height of $2.14 \mathrm{~m}$. The slope gradients, found using linear regression, are $-36.75 \mathrm{~dB} /$ decade, and $-38.5 \mathrm{~dB} /$ decade for stand-offs of $1 \mathrm{~mm}$ and $10 \mathrm{~mm}$ respectively, indicating that we have achieved a good performance with both antenna configurations. We note that for greater receiver antenna heights, the transition to pure $4^{\text {th }}$ order propagation does not occur until larger antenna separations, and we observe in our measurements that the best match to the theoretical result (i.e., closest to $-40 \mathrm{~dB} /$ decade slope) is achieved at the lowest receiver antenna height, i.e., $2.14 \mathrm{~m}$. (for comparison, Figure 8 shows the path loss as a function of distance for the receiver height of $6.29 \mathrm{~m}$, which has a gradient of $24.8 \mathrm{db} /$ decade and $22.1 \mathrm{~dB} /$ decade for stand-offs of $1 \mathrm{~mm}$ and $10 \mathrm{~mm}$ respectively).

As well as verifying that our antenna configuration has a reasonable agreement with the expected theoretical results, we can also compare the received signal power to that measured in the previous FH WSN deployment, where the antenna was located in the FH chamber itself. We compare our results to those presented in [ [1] Figure 7 '2m Antenna Height North'], observing 

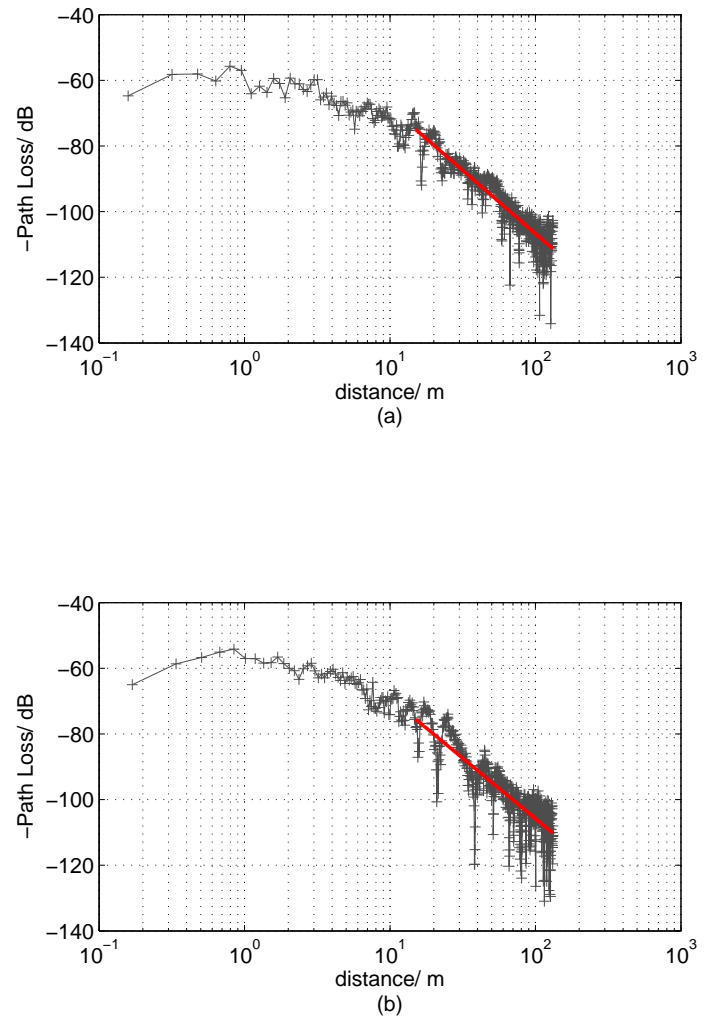

Figure 7: (a) $2.14 \mathrm{~m}$ receive antenna height, $1 \mathrm{~mm}$ stand-off between transmitter and ground plane; (b) $2.14 \mathrm{~m}$ receive antenna height, $10 \mathrm{~mm}$ stand-off between transmitter and ground plane.

that we have obtained a reduction in path loss of approximately $10 \mathrm{~dB}$. Note the subsequent measurements [15] show that the path loss with the antenna located in the FH chamber may be even greater than that given in [1], therefore we can say that we have achieved a path loss reduction of at least $10 \mathrm{~dB}$. In [1], it is shown that a transmit power of $19 \mathrm{dBm}$ would be required to achieve their specified range of $73 \mathrm{~m}-100 \mathrm{~m}$. Using the low-profile antenna could potentially lead to a reduction in transmission power of $10 \mathrm{dBm}$, which would significantly reduce power consumption, and enable a wider variety of wireless sensor motes to be used. 


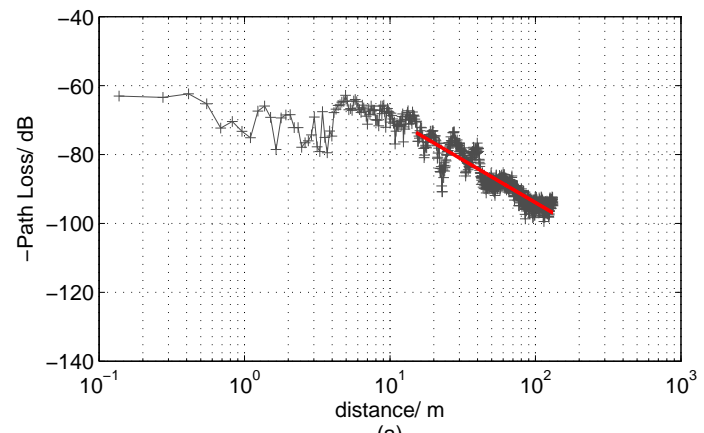

(a)

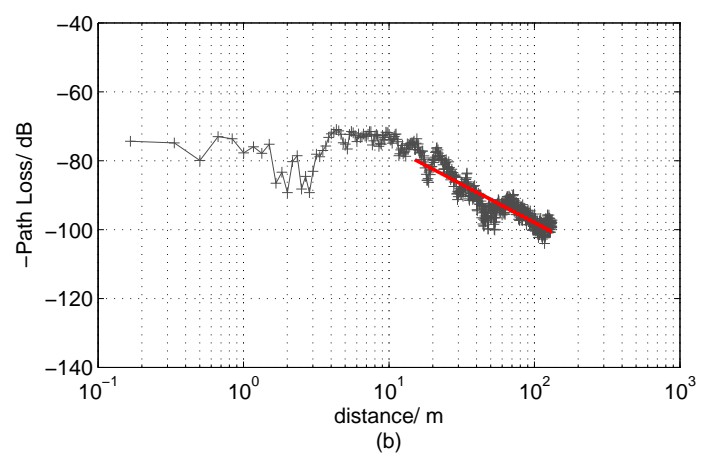

Figure 8: (a) $6.29 \mathrm{~m}$ receive antenna height, $1 \mathrm{~mm}$ stand-off between transmitter and ground plane; (b) $6.29 \mathrm{~m}$ receive antenna height, $10 \mathrm{~mm}$ stand-off between transmitter and ground plane.

\section{Conclusion}

In this paper we have assessed the performance of a compact low-cost, low profile planar antenna for operation in the $2.4 \mathrm{GHz}$ ISM band, to be deployed in WSNs monitoring the water supply at FHs. We have confirmed that this antenna has the required properties of an omnidirection radiation pattern and high efficiency. Channel characterisation measurements with the antenna deployed in a FH demonstrate its suitability for such applications. Furthermore we have shown that a reduction in path loss of at least $10 \mathrm{~dB}$ is achieved compared to the previously arrangement, where the WSN antenna was located in the below ground FH chamber. Having demonstrated a 
proof of concept, further work is now required to robustly integrate the antenna as part of the FH lid.

\section{References}

[1] M. Lin, Y. Wu, and I. Wassell, "Wireless sensor network: Water distribution monitoring system," in Radio and Wireless Symposium, 2008 IEEE, jan. 2008, pp. 775 -778.

[2] S.-C. Hsia, S.-W. Hsu, and Y.-J. Chang, "Remote monitoring and smart sensing for water meter system and leakage detection," Wireless Sensor Systems, IET, vol. 2, no. 4, pp. 402408, December 2012.

[3] A. Garcia, T. Tose, L. Ramalho, and D. Sicari, "Wireless sensor networks zigbee applied on sewage treatment station," in Wireless Sensor Systems (WSS 2012), IET Conference on, June 2012, pp. 1-5.

[4] "IEEE Standard 802.15.4." [Online]. Available: http://standards.ieee.org/findstds/ standard/802.15.4-2011.html

[5] "Memsic MICAz." [Online]. Available: http://www.memsic.com/wireless-sensor-networks/

[6] C. Delaveaud, P. Leveque, and B. Jecko, "New kind of microstrip antenna: the monopolar wire-patch antenna," Electronics Letters, vol. 30, no. 1, pp. 1 -2, jan 1994.

[7] G. Conway, W. Scanlon, and D. Linton, "Low-profile microstrip patch antenna for over-body surface communication at $2.45 \mathrm{GHz}$," in Vehicular Technology Conference, 200\%. VTC2007Spring. IEEE 65th, april 2007, pp. $392-396$.

[8] G. Conway, W. Scanlon, C. Orlenius, and C. Walker, "In situ measurement of UHF wearable antenna radiation efficiency using a reverberation chamber," Antennas and Wireless Propagation Letters, IEEE, vol. 7, pp. $271-274,2008$.

[9] A. Chandran, G. Conway, and W. Scanlon, "Compact slot-loaded patch antenna for 868 MHz wireless body area networks," in Antennas and Propagation Conference, 2008. LAPC 2008. Loughborough, march 2008, pp. $433-436$. 
[10] T. Loh, M. Alexander, F. Widmer, P. Miller, and D. Knight, "Validation of a new smallantenna radiated testing range," in Antennas and Propagation, 2009. EuCAP 2009. 3rd European Conference on, march 2009, pp. $699-703$.

[11] T. H. Loh and M. Alexander, "New facility for minimally invasive measurements of electrically small antennas," in Antennas and Propagation Conference, 2008. LAPC 2008. Loughborough, march 2008, pp. $313-316$.

[12] "CST microwave studio." [Online]. Available: www.cst.com

[13] T. S. Bird, "Definition and misuse of return loss [report of the transactions editor-in-chief]," Antennas and Propagation Magazine, IEEE, vol. 51, no. 2, pp. 166-167, 2009.

[14] J. D. Parsons, The Mobile Propagation Channel. John Wiley and Sons., 2000.

[15] M. Lin, "Channel modelling for wireless sensor networks," Ph.D. dissertation, Computer Laboratory, University of Cambridge, 2009. 